\title{
Gesundes Essen erhält die Kognition im Alter
}

\section{Bei älteren Patienten mit vaskulären Erkrankungen führt eine gesunde Ernährung zu einer geringeren Rate kognitiver Einschränkungen.}

_ Für eine Studie wurden
Daten der beiden großen
Endpunktstudien ON-
TARGET und TRANS-
CEND mit Patienten über
55 Jahren mit vaskulären
Erkrankungen ausge-
wertet. Erstere untersuchte eine Monothera-

\section{Hier steht eine Anzeige.}

pie mit Telmisartan im

Vergleich zu einer Kombination mit Ramipril [N Engl J Med. 2008;358:1547-59], letztere eine Therapie mit Telmisartan für Patienten, die ACE-Hemmer nicht tolerierten [Lancet. 2008;372:1174-83]. Zu Beginn erhielten 27.860 Studienteilnehmer eine Mini Mental State Examination (MMSE) und das Food Frequency Questionnaire, das u. a. den Konsum von Gemüse, Obst, tiefgefrorenen Nahrungsmitteln, Fisch, Fleisch, Eiern und Alkohol erhebt. Diese Daten wurden in einen modifizierten Alternative Healthy Eating Index (mAHEI) transformiert. Nach 4,9 Jahren wurde eine weitere MMSE durchgeführt. Eine Abnahme der kognitiven Leistungen war definiert als eine Reduktion von drei oder mehr Punkten.

Die Patienten wurden dann nach ihrem mAHEI-Scores in fünf Gruppen eingeteilt. Teilnehmer mit gesunder Ernährung waren älter, körperlich aktiver, schlanker, rauchten seltener und hatten höhere MMSE-Werte. Eine Abnahme der kognitiven Funktionen wurde bei 4.699 Teilnehmern (16,8\%) beobachtet. Im Rahmen einer multivariablen Analyse ergab sich eine inverse Assoziation zwischen der Qualität der Ernährung und dem Risiko einer Abnahme kognitiver Funktionen. Wurden die Teilnehmer mit der gesündesten und der ungesündesten Ernährung miteinander verglichen, ergab sich eine Risikoreduktion um den Faktor 0,76, die statistisch signifikant war.

- Smyth A, Dehghan M, O'Donnell M et al. Healthy eating and reduced risk of cognitive decline: A cohort from 40 countries. Neurology. 2015;84:2258-65

\section{KOMMENTAR}

Der eindeutige Zusammenhang zwischen gesunder Ernährung und kognitiven Funktionen im Alter ist ein wichtiges Nebenergebnis der beiden großen Studien. Die Behandlung von vaskulären Risikofaktoren per gesunder Ernährung beeinflusst sehr wahrscheinlich nicht nur das Auftreten und Fortschreiten der vaskulären, sondern auch der degenerativen Demenz positiv. Aufklärungs- und Schulungskampagnen zur gesunden Ernährung können hier präventiv wirksam sein. 